\title{
Controversias de la Guerra Fría cultural. Una reflexión desde los American Studies, 1945-1975
}

\author{
Francisco J. RODRÍGUEZ JIMÉNEZ \\ Universidad de Salamanca \\ moralito@usal.es
}

Recepción: 16 de febrero de 2010 / Revisión: 24 de mayo de 2010

Aceptación: 2 de julio de 2010 / Publicación: diciembre de 2010

\begin{abstract}
RESUMEN
Con el inicio de la Guerra Fría se desencadenó también una batalla cultural entre soviéticos y estadounidenses. El propósito de unos y otros fue irradiar sus respectivos modelos socio-culturales e ideologías en el campo rival y, sobre todo, frenar la expansión de los valores contrarios en el propio. La promoción y difusión de los Estudios Norteamericanos o American Studies en las universidades del bloque europeo occidental formó parte de aquel conflicto. Poco sabemos por el momento acerca de quién, o quiénes, y con qué medios intentaron que la Historia, el Arte, la Literatura o la Ciencia Política estadounidenses fuesen mejor conocidos por los ciudadanos del viejo continente. En las páginas que siguen pretendemos arrojar algo de luz sobre esos interrogantes. Simultáneamente y desde la perspectiva de lo sucedido en torno a los American Studies, se aportarán datos e indicios para la reflexión sobre cuestiones del contexto general de la Guerra Fría.
\end{abstract}

Palabras clave: Guerra Fría cultural, proselitismo cultural, propaganda, American Studies Movement, siglo XX.

\section{Controversies of the Cultural Cold War. A reflection on the American Studies, 1945-1975}

\begin{abstract}
The Cold War also triggered a cultural battle between the Soviets and U.S. Americans. Both aimed to radiate their own social and cultural models, together with their own ideologies, in their rival's sphere of influence, and above all, to check the expansion of their opponent's values in their own sphere. The promotion and diffusion of U.S. or American Studies in the universities of the Western European block was part of that conflict. To date, little is known about the people who tried to improve European appreciation of U.S. History, Art, Literature or Political Science, or about the means they used. This article will also discuss more general issues of the cultural cold war, such as: When did the U.S. propaganda machine start to function? or Did the Soviet Union invest more in propaganda than the United States?

Keywords: Cultural Cold War, Cultural Proselytism, Propaganda, American Studies Movement, XXth Century.

SUMARIO: 1. Introducción. 2. Un nuevo campo de estudios. 3. ¿Cuándo echó a rodar la maquinaria de propaganda cultural americana? 4. Discrepancias sobre la conveniencia de invertir en proselitismo cultural. 5. ¿Cuánto gastaron unos y otros? 6. Esfuerzos privados en pro de los American Studies. 7. Referencias bibliográficas.
\end{abstract}




\section{INTRODUCCIÓN ${ }^{1}$}

El desembarco de Normandía y las consecuencias político-estratégicas y económicas que trajo aparejadas son temas ampliamente abordados desde el punto de vista historiográfico ${ }^{2}$. Hubo, sin embargo, otro desembarco más discreto: el de la cultura estadounidense a esta orilla del Atlántico. Sobre este último quedan todavía muchos interrogantes por resolver.

Si bien en momentos históricos anteriores la cultura no quedó completamente al margen del enfrentamiento entre los distintos estados ${ }^{3}$, con la Guerra Fría se intensificó el empeño de los distintos gobiernos por utilizarla con fines geopolíticos. El uso de la cultura como arma propagandística durante el periodo de enfrentamiento bipolar ha sido objeto ya de algunos trabajos ${ }^{4}$; no obstante, probablemente sea una de las facetas menos estudiadas de aquel conflicto. Una cierta intangibilidad ha acompañado a gran parte de las iniciativas institucionales emprendidas en ese terreno, carácter escurridizo que ha hecho que, en algunas ocasiones, hayan pasado desapercibidas. Ahí radica su potencialidad a la hora de utilizarla como "arma propagandística", al tiempo que es el motivo por el que su estudio puede resultar complicado.

Partiendo de esas premisas, no es raro encontrar referencias escritas o declaraciones sobre la supuesta implicación del gobierno estadounidense en la promoción de los Estudios Norteamericanos o American Studies en el extranjero. Siguiendo tal planteamiento, Washington habría incentivado la difusión de dichos estudios en el bloque europeo occidental, como parte de sus esfuerzos propagandísticos en el frente cultural contra Moscú. Por el momento, tales afirmaciones se han basado fundamentalmente en algunos testimonios personales 5 e indicios dispersos ${ }^{6}$ más que en una apoyatura documental sólida ${ }^{7}$.

${ }^{1}$ El marco cronológico tomado como referencia es 1945-75. La razón de tal elección tiene que ver con la dificultad, a veces imposibilidad, de acceder a la documentación posterior. Algunas de las siglas que emplearemos son: BFS: Board of Foreign Schoolarship; ACFE: Archivo de la Comisión FulbrightEspaña; FFA: Ford Foundation Archive; NARA: National Archives and Records Administration; LC: Library of the Congress.

2 De la amplia literatura existente y sin ánimo de exhaustividad se podrían citar las obras de Soutou, 2001; MAIER, 1996 o LefFler - PAINTER, 1994.

3 NiÑo, 2009.

${ }^{4}$ La dimensión cultural de aquel enfrentamiento es un terreno todavía menos explorado. Algunos de los trabajos más recientes son: León, 2010; Delgado, 2009; ScotT-SMith, 2008 o HiLton - MinNen, 2007.

5 Pells, 1997, pp. XIV y 61.

${ }^{6}$ Hay quien ha afirmado, sin demasiada apoyatura documental, que "American Studies, of course, has had a particularly dramatic entanglement with public diplomacy and the Cold War contest for "hearts and minds", and legacies of that entanglement still haunt the field imaginary today". KeNNEDY - LUCAS, 2005, pp. 309-310.

7 Probablemente, la primera incursión en este ámbito historiográfico sea el libro de SKARD, 1958. Sin embargo, dicha obra presenta numerosas carencias ya que no cuestiona el nexo entre los esfuerzos por promocionar aquellos estudios y el contexto de Guerra Fría cultural en que se dieron. Tampoco aporta fuentes documentales que expliciten aquella conexión. Skard, convencido atlantista, se limita a narrar la evolución de los American Studies en los distintos países europeos tomados como referencia. Más reciente en el tiempo, la obra de Scoтt-Sмiтh, 2007, va más allá y sí adopta un enfoque crítico, si bien -tal vez por la limitación espacial propia de un artículo- deja en el aire muchos interrogantes. 
El presente artículo pretende demostrar que efectivamente los distintos ejecutivos estadounidenses se interesaron porque sus Humanidades y Ciencias Sociales fueran conocidas y entendidas por los europeos, y valorados como productos de calidad e independientes de la British Culture. El objetivo: coadyuvar a fortalecer el consenso entre las élites europeas sobre la conveniencia de asumir el liderazgo americano del mundo occidental.

Sucede que, al tiempo que tratábamos de corroborar la hipótesis antedicha ${ }^{8}$, fueron surgiendo interrogantes que no quedaban del todo claros en parte de la literatura existente. Nos centraremos en varios de ellos: ¿cuándo empezó la implicación gubernamental de los Estados Unidos en la Guerra Fría cultural?, ¿fue únicamente a la defensiva, recogiendo el guante soviético como han dejado entrever algunos autores? Es conveniente también preguntarse si la relación entre universidades, multinacionales y fundaciones filantrópicas estadounidenses y el gobierno de aquel país en la tarea de fomentar los American Studies fue impuesta por parte gubernamental, o bien se trató de una suerte de "simbiosis" o comunidad de intereses forjada al socaire del temor común a la amenaza comunista.

Este último punto nos lleva a su vez a plantearnos si es correcto afirmar que los soviéticos gastaron mucho más en propaganda cultural que los americanos. Si bien es pronto para conclusiones definitivas, se puede adelantar que hay datos, al menos en lo que se refiere a la promoción de los American Studies, que hasta el momento han sido pasados por alto. Y es que hasta ahora no se han tenido en cuenta las importantes sumas de dinero que se canalizaron para el sostenimiento y consolidación de este conjunto de estudios a través de las vías privadas antedichas. Esa inversión quedó al margen de las estadísticas oficiales. Así pues, pudiera ser que la balanza final no estuviese tan inclinada del lado comunista como se ha afirmado.

\section{UN NUEVO CAMPO DE ESTUDIOS}

El planteamiento de partida era que bajo la etiqueta de American Studies o American Civilization se consolidase un área de estudios heterogénea e interdisciplinar dedicada al análisis de los aspectos socio-culturales de la sociedad norteamericana. Uno de los primeros planes para la difusión de este tipo de estudios en el exterior fue expuesto en los términos siguientes:

The courses and subject-matter areas considered to fall within the field of American Studies include: American history, American literature and civilization, American education, American government, American architecture and the arts, political and social sciences $[. .$.$] , the teaching of English by Americans or American trained teachers and$ other subjects when taught as part of an area study specialization dealing principally with the United States ${ }^{10}$.

\footnotetext{
8 RodrÍGUEZ, 2010.

9 Berghahn, 1999, p. 394.

10 American Studies inventory and survey. Washington, D.C., 29-VII-1955. NARA RG 59, BFSPlans and Development, 1955-60, box 45.
} 
Se trataba, por tanto, de un plan ambicioso que pretendía que aquellas disciplinas gozasen de un mayor grado de autonomía; y que a su vez dejasen de ser un apéndice de otras asignaturas de carácter más general, articuladas desde un punto de vista eurocéntrico, fundamentalmente británico.

No han faltado las críticas a la categoría de American Studies, puesto que este término encierra una cierta simplificación de la realidad: "lo americano" es mucho más rico, complejo y amplio de que lo se pueda producir tan sólo en el país de Lincoln y Jefferson. Quienes así piensan han optado en las últimas décadas por un término, a su juicio, más adecuado: USAmerican Studies ${ }^{11}$. Se separa de este modo lo que es propio de la nación estadounidense de lo que puede ser también norteamericano, pero no estadounidense, o, por qué no, centroamericano, sudamericano o iberoamericano.

Pese a las cautelas mencionadas, optaremos aquí por seguir usándolo, ya que fue el término más utilizado por los agentes de la diplomacia cultural al servicio de Washington en el periodo tomado como referencia. Fue el más habitual, aunque no el único. También se emplearon los de American Civilization o el todavía más genérico de American Culture.

En una primera fase, allá por las primeras décadas del siglo XX, cuando estas disciplinas apenas habían comenzado a contar con espacio propio en los planes de estudio universitarios estadounidenses, las denominaciones antedichas fueron, a veces, usadas indistintamente como sinónimos. Situación que produjo algunos problemas a la hora de determinar si algunas asignaturas, borderlines, debían o no incluirse en los planes de proselitismo cultural en el extranjero. El caso más notorio en este sentido fue el de disciplinas del ámbito de las Ciencias Sociales, como la Sociología y la Ciencia Política. Para algunos de los promotores gubernamentales de los American Studies, estas asignaturas debían formar parte de aquellos. Pero no todos eran de la misma opinión, ni dentro del engranaje diplomático ni entre los profesionales, generalmente del mundo universitario, dedicados a su estudio ${ }^{12}$.

Diversos han sido los factores que han determinado que los planes institucionales se materializasen, en algún caso según estaba previsto, en otros tan sólo a medias, y poco o nada en el resto. La situación inicial no era nada prometedora. Los American Studies tuvieron que superar numerosas dificultades antes de ser considerados como un campo de estudios autónomo y de calidad dentro del mundo académico europeo ${ }^{13}$. Los factores coadyuvantes y los que lo han dificultado son varios:

- Diferencia de criterios políticos entre la gran potencia y los gobiernos europeos que debían darles la bienvenida.

11 Hilton - Van Minnen, 2007, p. 9.

12 American Studies inventory and survey. Washington, D.C., 29-VII-1955. NARA RG 59, BFSPlans and Development, 1955-60, box 45. Aparte del documento antedicho, véase también SPILLER, 1973, pp. 611-618. En realidad, la polémica sobre qué asignaturas debían incluirse dentro de los American Studies y cuáles no continuó más allá de los momentos iniciales y se aprecia todavía en momentos recientes. VERHEUL, 1999, pp. 91-103.

13 De hecho, todavía siguen en una situación de precariedad en no pocos países. Véase una buena panorámica sobre la salud de la enseñanza de la Historia de los Estados Unidos en Europa, uno de los pilares fundamentales de los American Studies, en Hitton - Van Minnen, 2007. 
- La percepción de la ciudadanía respecto al papel que Estados Unidos desempeñaban en el orden internacional.

- El interés del mundo universitario por ampliar el conocimiento de lo que ocurría dentro y en torno a la nación americana ${ }^{14}$.

- El modo en que los profesionales dedicados a su estudio se han adaptado a las cambiantes oportunidades curriculares y a las tendencias, debates y paradigmas existentes ${ }^{15}$.

La inmensa mayoría de artículos o libros que se han ocupado de los American Studies lo han hecho para analizar las obras compuestas bajo esta denominación, las tendencias, los debates, los paradigmas ${ }^{16}$, etc., pero no los entresijos de la implicación del gobierno de Estados Unidos por potenciarlos y utilizarlos como instrumento propagandístico en su enfrentamiento con la URSS.

\section{3. ¿CUÁNDO ECHÓ A RODAR LA MAQUINARIA DE PROPAGANDA CULTURAL AMERICANA?}

$\mathrm{Al}$ menos en lo que se refiere a fomentar la proyección exterior de los American Studies, el punto de partida podría situarse entre mediados y finales de la década de los años cuarenta, en un contexto caracterizado por el incremento de la tensión con la Unión Soviética. El documento más antiguo que hemos localizado al respecto está fechado en 194917 .

Parece, sin embargo, que no fue el primero. Los servicios diplomáticos estadounidenses llevaban ya algún tiempo trabajando sobre este particular. Creemos que es el caso de otro memorándum posiblemente más antiguo ${ }^{18}$. Pese a que no se indica cuándo fue redactado, de su lectura se colige que el ejecutivo de Estados Unidos estaba preocupado por el grado de desarrollo de los programas de American Studies en las universidades del propio país; también por las dificultades que se estaban encontrando a la hora de impulsar estos estudios en el extranjero desde hacía unos

14 Generalmente el interés del alumnado y el del profesorado han ido por caminos diferentes.

15 Este factor junto con las motivaciones, preferencias, e intereses de las autoridades educativas de los distintos países de acogida habrían determinado la suerte de aquellas disciplinas en mayor medida de lo mucho o poco llevado a cabo desde medios institucionales norteamericanos por potenciarlas, véase: HiLTON - VAN MinNEN, 2007, p. 44. Dicho razonamiento ya fue apuntado por Skard en los años sesenta: "But the decisive factors will always be in Europe, as they were in the past: in European attitudes and opinions toward the U.S.A in the strength of realism and enlightened self-interest in European minds". Véase: SKARD, 1958, p. 653.

16 Sobre los primeros compases y formulaciones teóricas en torno a los American Studies pueden consultarse: VERHEUL, 1999 O MAY, 1996.

17 The Nature and Implications of Programs in American Civilization. Washington, D.C., 18-XI1949. NARA RG 59, BPA, Correspondence, Memorandums, etc., 1951-56, box 9.

18 Investigation of Foreign Programs for Instruction in American Civilization. Washington, D.C. (doc. s. f.) NARA RG 59, BFS- Plans and Development, 1955-60, box 43. 
años. Se citan algunos datos que permiten pensar que se realizó en algún momento de 1947 , tal vez $1946^{19}$, pero también es probable que exista documentación anterior.

¿Por qué se produjo la implicación del gobierno norteamericano para potenciar institucionalmente un campo de estudios que hasta entonces había caminado básicamente por impulso privado? Debemos recordar, aunque sea sucintamente, que el apoyo principal para el crecimiento y consolidación de los American Studies venía dándose por parte de instituciones y personajes de la sociedad civil estadounidense desde las primeras décadas del siglo XX. Por entonces, el American Studies Movement caminaba sin apenas injerencia gubernamental ${ }^{20}$.

Algunos autores, como por ejemplo Frances Stonor, han señalado que una implicación total por parte estadounidense en la utilización de la cultura como instrumento propagandístico se produjo más tarde; en torno a mediados de la década de los cincuenta, de la mano de la campaña psicológica "para ganar las mentes y las voluntades de los hombres"21, lanzada durante la presidencia de Eisenhower.

Por su parte, Jessica Gienow-Hecht ha mantenido que en los primeros compases de los años cincuenta el gobierno estadounidense no acabó de intervenir explícitamente y de manera decidida en la batalla cultural contra el comunismo. Sostiene que fueron determinados intelectuales y profesores de aquel país, "cold war warriors" los que tuvieron que "tirar del carro", pidiendo una mayor implicación al respecto por parte de Washington 22 .

Interpretaciones de este tipo son también barajadas por Joël Kotek ${ }^{23}$. De las mismas parece colegirse la idea de que Estados Unidos fue a la zaga de la URSS en materia de financiación de actividades propagandísticas en el exterior durante el período antedicho, también que cuando se actuó en tal sentido se hizo más bien como respuesta a la orquestada batería de medidas para denigrar el nombre de Estados Unidos que estaba llevando a cabo -desde Washington se argumentaba que con bastante anterioridad- la Unión Soviética. Siguiendo esa lógica, los norteamericanos se habrían limitado a recoger el guante del desafío.

En tal contexto interpretativo, la agresión norcoreana a su vecino del sur, en junio de 1950, habría sorprendido a los americanos. No fue más que una coincidencia, se

${ }^{19}$ No olvidemos que en el plano geopolítico la tensión entre soviéticos y norteamericanos había ido en aumento incluso desde antes de la rendición alemana. La actitud menos "apaciguadora" de Truman hacia Stalin en Postdam, el denominado "largo telegrama" de febrero de 1946 en el que George Kennan advertía de los propósitos expansionistas de Moscú y suscitaba la necesidad de implementar una política de contención del comunismo, o el discurso de Churchill en Fulton sobre el telón de acero son buena prueba de ello.

${ }^{20}$ Más detalles sobre la aparición, evolución y naturaleza del American Studies Movement como movimiento de la sociedad civil estadounidense en pro de la reivindicación de una American Culture de calidad e independiente del sambenito de ser subproducto de la British Culture en el apartado: "American Studies para un mundo bipolar" en RodRíGUEZ JiMÉnEZ, 2010, pp. 29 y ss.

21 STONOR, 2001, p. 56.

22 "The world, in short, needed more American culture, and it was the government's job to provide it". Véase: GienOw - Hecht, 2000, p. 469.

23 Kотек, 2003. Una visión mucho más crítica respecto a la implicación de Washington en este tipo de "dirty business" es la ofrecida por SNOw, 2002. 
intentó difundir desde medios gubernamentales estadounidenses ${ }^{24}$, que aquel ataque tuviese lugar el mismo día que se inauguraba el Congreso por la Libertad de la Cultura -financiado secretamente por la CIA-en el sector de la capital alemana bajo control norteamericano:

On the day the delegates assembled in Berlin, Communist forces in North Korea invaded the non-Communist Republic of Korea. This coincidence focused world attention on the reactions of such celebrated figures as Arthur Koestler and James Burnham ${ }^{25}$.

La coincidencia entre ambos eventos hizo que las declaraciones de condena a la participación soviética en Corea, pronunciadas por parte de los intelectuales europeos reunidos en Berlín, tuvieran mayor eco mediático. Y es que aquellas críticas serían realizadas a título personal y sin ninguna vinculación aparente con la Casa Blanca, lo que en buena lógica reforzaba su credibilidad.

¿Fue realmente una casualidad? Analizando el dossier The Congress for Cultural Freedom del que procede la cita anterior, parece que aquella situación, si bien puede que casual en cuanto al momento último de desencadenarse, había sido largamente esperada y planificada. Desde Washington se habían dado órdenes para que se cubriese informativamente todo lo que se pudiese producir en la ciudad alemana. Había que estar al tanto por si en algún momento se podía sacar partido propagandístico. Así quedaba reflejado en un apartado del informe confidencial mencionado:

The Department had decided months in advance that the Congress should be given full coverege, and that all information media should report on the Congress with heavy play ${ }^{26}$.

La sorpresa quizá existió en cuanto al momento concreto en que se desencadenó la Guerra de Corea, no en cambio sobre la repercusión mediática y el rédito político que se esperaba obtener de las jornadas organizadas en Berlín en la lucha contra Moscú.

Es difícil de precisar en qué medida la cobertura mediática fue ejercida libremente por los medios europeos o si respondió a presiones de instancias gubernamentales norteamericanas. Lo cierto es que los servicios de información y propaganda de Washington no estaban en estado de hibernación, como dan a entender algunas de las obras mencionadas líneas atrás. Estaban actuando y lo hacían desde hacía tiempo.

¿Por qué no se admitió abiertamente la financiación del Congreso por la Libertad de la Cultura con dinero público estadounidense desde un primer momento, como sí hicieron los soviéticos con el Congreso de Escritores de Berlín Oriental, celebrado en el teatro Kammerspiel a finales de $1947 ?^{27}$

24 The Congress for Cultural Freedom. Berlín, 26-VI-1950. NARA RG 59, Bureau of Public Affairs, 1944-62, box 67.

25 Ibídem. Las cursivas de la cita son nuestras.

26 Ibídem.

27 La organización de eventos culturales por parte de Moscú venía de largo; ya en 1932 el Komintern había auspiciado la celebración de un Congreso Internacional de escritores contra la Guerra en Amsterdam. También célebres fueron las citas del Congreso en Defensa de la Cultura, celebrado por primera vez en París en 1935 y presidido por André Gide y André Malraux. Encuentro que tendría su 
Un elemento que estará presente no sólo en estos momentos iniciales de la partida cultural contra los comunistas, sino también en lo sucesivo ${ }^{28}$ fue el intento del gobierno americano de no aparecer implicado en actividades propagandísticas. De hecho, ni siquiera se utilizaba abiertamente el término propaganda ${ }^{29}$. Las más de las veces se usaron numerosos circunloquios o perífrasis con tal de evitar aquella palabra tabú. Se decía que los Estados Unidos no se ponían al mismo nivel que la URSS y que, por tanto, no utilizaban los mismos métodos. Su juego era más limpio, más democrático...

Por lo demás, convendría no dejar de contemplar otra posible interpretación. Y es que tales reticencias por parte del ejecutivo estadounidense pudieron responder a un modus operandi asumido conscientemente: evitar que una implicación más explícita en aquel dirty business desatase las críticas de la opinión pública interna. Este temor a aparecer implicados en aquel "juego sucio" no se produjo únicamente en los compases iniciales de la Guerra Fría. Por el contrario, se repetirá en las décadas siguientes ${ }^{30}$. No en vano hay quien ha afirmado que los Estados Unidos usaron la cultura con fines propagandísticos, pero como con mala conciencia de estar haciéndolo31; o bien que fueron "propagandistas reticentes"32 durante toda la Guerra Fría.

En este sentido, resulta significativo lo que se decía en un informe confidencial de los agentes de la diplomacia cultural norteamericana destinados en España en los años sesenta. Dicho memorándum mencionaba las distintas estrategias de captación de élites y de persuasión de los estudiantes universitarios españoles que se estaban ejecutando. De entre ellas, la promoción y difusión de los Estudios Norteamericanos ocupaba un lugar destacado. Sin embargo, aquel proselitismo cultural tenía un valor añadido, una "plusvalía" que no se podía hacer explícita. Por eso se actuaba "por debajo de la mesa":

In Spain, because of regime sensitivity to contacts with opposition, or even under certain circumstances with university students, USIS diffuses this objective [evolution-

continuidad en el II Congreso Internacional de Escritores para la Defensa de la Cultura, celebrado en 1937 en la España de la guerra civil. No menos importante fue la red de "Asociaciones de Amigos de la Unión Soviética", que venía tejiéndose desde los años veinte del pasado siglo. Y es que la utilización del término 'propaganda' no tenía por entonces, y sobre todo en el ámbito soviético, las connotaciones negativas que sí tendría posteriormente en los discursos estadounidenses. Más detalles sobre las claves del funcionamiento de la propaganda cultural soviética en ZASLAVSKY, 2004, pp., 85-106.

28 Precisamente una de las conclusiones de un informe de los servicios diplomáticos estadounidenses de 1975 señalaba la ambigüedad que había existido en torno al uso de ese término. Una confusión, se apuntaba, que había generado complicaciones internas. Véase: The United States Communicates with the World: A Study of U.S International Information and Cultural Programs and Activities. Washington, D.C., 25-VIII-1975. NARA RG 306, Post Publications, 1953-99, box 65.

29 Tampoco es que aparezca muy a menudo en la documentación confidencial manejada.

30 Véase: The United States Communicates with the World: A Study of U.S International Information and Cultural Programs and Activities. Washington, D.C., 25-VIII-1975. NARA RG 306, Post Publications, 1953-99, box 65.

31 "Americans have never felt fully at ease with cultural diplomacy activities [...] both because of a widespread belief that diplomacy is about power (rather than mutual interest) and because of an aversion to 'official culture'. Véase: NinKOVICH, 1996, pp. 5-7.

32 Gienow-Hecht, 1999, p. 5. 
ary progress toward more democratic political processes] under the rubric, "American Studies», covering our supporting informational and cultural activities across-the-board ${ }^{33}$.

\section{DISCREPANCIAS SOBRE LA CONVENIENCIA DE INVERTIR EN PROSELITISMO CULTURAL}

Pese a lo mantenido por ciertas interpretaciones maniqueas respecto a la Guerra Fría $^{34}$, ni entre la clase política estadounidense, ni entre su sociedad civil existió consenso sobre la conveniencia o no de invertir en este tipo de actividades de propaganda cultural. Tampoco en el caso de hacerlo en los términos de la intervención. Eran muchos los que en el interior de Estados Unidos no sólo no creían en la potencialidad del factor cultural en la arena internacional, sino que desconfiaban abiertamente de aquel tipo de iniciativas ${ }^{35}$.

Diversos círculos políticos estadounidenses, especialmente los más conservadores, miraban con recelo ciertas manifestaciones culturales en sí mismas. Así por ejemplo algunas líneas creativas y de expresión artística ${ }^{36}$ del grupo de los American Studies, sobre todo en el terreno de la vanguardia pictórica y literaria, fueron duramente criticadas ${ }^{37}$. Un clima, pues, poco alentador para quienes por entonces se dedicaban al cultivo de ciertas facetas de los Estudios Norteamericanos. Mal se podían proyectar hacia fuera, cuando dentro no había consenso sobre su validez o sobre si eran o no peligrosas para los intereses nacionales.

La sociedad norteamericana vivía por entonces un fuerte debate interno. El arco político se encontraba bastante polarizado. Por un lado, los "aislacionistas-militaris-

33 Annual Assesment Report for Spain for 1965. Washington, D.C., 27-XII-1965. NARA RG 306, Country Exhibits, 1955-67, box 29. Las cursivas son nuestras.

34 Un buen ejemplo de ésas interpretaciones que veían a los servicios diplomáticos americanos operando siempre al unísono y como una maquinaria perfectamente engrasada de propagación e inoculación del American Way of life es la obra VÁZQUEZ, 1973. No es que no hubiera propósito de captación y persuasión, sino que la realidad tuvo más matices y no se puede limitar a análisis reduccionistas.

35 Tales renuencias no se dieron únicamente en los compases iniciales de la Guerra Fría. Por el contrario, se mantuvieron en parámetros similares durante las décadas siguientes. Véase, a modo de ejemplo, lo que se decía al respecto a mediados de los años sesenta: A report on the strategic importance of Western Europe. Washington, D.C., 24-IX-1964. NARA RG 59, General Records of BFS, 1950-70, box 19. Una década más tarde encontramos valoraciones similares en el informe: The United States Communicates with the World: A Study of U.S International Information and Cultural Programs and Activities. Washington, D.C., 25-VIII-1975. NARA RG 306, Post Publications, 1953-99, box 65.

36 Sobre el componente "revolucionario", de ruptura con los patrones metodológicos y temáticos de las enseñanzas universitarias tradicionales que rodeó los primeros pasos en pro de la institucionalización de los American Studies, véase: MARX, 1979.

37 En los círculos políticos más conservadores surgieron grandes recelos hacia aquellos artistas de estilo más rompedor. Se les atribuían -a veces con razón- tendencias políticas de izquierda, cuando no directamente comunistas. Esto creó la situación paradójica de que se celebraron exhibiciones con sus obras fuera de Estados Unidos, mientras que se tuvieron que cerrar o clausurar algunas de las proyectadas en el propio país por miedo a topar con la "inquisición cultural" que el macarthismo desató. Véase: American Cultural Presentations Abroad. Washington, D.C., (doc. s. f). NARA RG 59, Activities in Foreign Countries, 1950-76, box 3255. 
tas" 38 , partidarios de actuar con firmeza y pragmatismo en la arena internacional, desdeñosos de la interacción cultural y el mutual understanding; por otro, quienes tenían un approach más sutil, más receptivo hacia las aportaciones en ese terreno del soft power ${ }^{39}$.

En opinión de estos últimos, buena parte de la culpa de que los Estados Unidos fuesen considerados en el extranjero como un pueblo materialista, sin cultura, ni interés por tenerla, estaba en casa. Eran aquellos tough guys aislacionistas y sus comentarios despectivos hacia el mundo del Arte y la Cultura los que estaban erosionando el prestigio de la nación y dando argumentos a los que desde fuera criticaban la "infancia artística" en que vivía todavía la gran potencia. Los nuevos tiempos no requerían más "John Waynes", sino gente instruida y preparada para comprender las claves de un mundo cada vez más pequeño. Sobre esta polémica, resulta especialmente revelador el testimonio de uno de aquellos intelectuales del bando "internacionalista-multilateralista", Henri Peyre, quien ya en 1952 se preguntaba: "Are Americans hated abroad?"40.

En su opinión, parte del desdén hacia la nación americana que se apreciaba entre buena parte de las élites del mundo de la cultura europea era alimentado continuamente por comentarios o situaciones que tenían lugar en el interior del propio país. Era la gasolina que hacía arder el fuego de ciertos comentarios antiamericanos pronunciados en el exterior:

Americans are a spiritual and a religious people in spite of their attachment to material goods, and mechanical implements affording greater leisure are certainly preferable to the squalor in which masses have to toil and live elsewhere. But the worst misconceptions about America have been fostered by Americans themselves. It is sadly true that this country suspects the word "culture", and that not only its politicians but its radio speakers, its business men, its executives fear to appear "effeminate" if they like music, painting, or literature.

Such an open scorn of culture, and even of brains, may have been somewhat justified in an earlier age, when conditions were primitive and the country needed "tough guys" and adventurers more than thinkers. But that age is now gone [....]. The problems to be solved require knowledge, scholarship, hard thinking, imagination, independence and originality of views. Its need is primarily for well trained minds ${ }^{41}$.

Con este tipo de ideas flotando en el ambiente ${ }^{42}$, había que ser cauto a la hora de financiar determinadas actividades culturales.

38 IKENBERRY, 2002.

39 Uno de los casos más señeros fue el del senador demócrata William Fulbright. Al frente de la Comisión de Exteriores del Senado de los Estados Unidos, fue una de las voces más críticas contra quienes defendieron una política exterior intervencionista y unilateral. Buena muestra de sus planteamientos respecto a la que debía ser la política exterior de su país los recogió en: FulBRIGHT, 1972.

40 Are Americans hated abroad? Washington, D.C., 9-II-1952. LC. ASA, Archives Part I: Administrative File, 1946-2003, box I: 111.

41 Ibídem, p. 5.

42 Las dificultades que sufrieron ciertos profesionales de las "artes liberales" dentro del propio país norteamericano en la década de los cincuenta son ampliamente descritas en: PeLLS, 1989. En ciertos cír- 
Los primeros compases del apoyo gubernamental a los American Studies se vieron mediatizado por aquel debate. Y no es de extrañar, puesto que lo habitual hasta el momento había sido que el gobierno invirtiese dinero con cuentagotas en un campo como el educativo-artístico, dejando la iniciativa a instituciones privadas.

La cuestión fue especialmente delicada en los años más duros del macarthismo. Algunas de las iniciativas en pro del fomento del mundo literario o artístico fueron consideradas atentatorias o subversivas de la moral establecida. Los agentes gubernamentales de la diplomacia cultural no se vieron libres de inculpaciones, sospechas e interrogatorios, y pronto se convirtieron en uno de los blancos favoritos del senador McCarthy.

Según Joël Kotek, esta situación hizo que en el interior del Departamento de Estado se operase con mucha prevención. Tanto aquel organismo como la CIA utilizaron screen foundations o tapaderas para camuflar que estaban financiando ciertas actividades de propaganda cultural en el viejo continente ${ }^{43}$.

Por lo demás, y antes de proyectar hacia el resto del mundo el valor de las Letras de Mr. Marshall, había que acabar de convencer a los que todavía andaban dubitativos dentro del propio país. La sociedad estadounidense vivía fragmentada al respecto. Una inmensa mayoría del pueblo norteamericano estaba convencido del poder de su economía, de su ejército y de su sistema político. Del valor de sus letras y sus artes no todos estaban tan seguros:

Why should Americans, who in the first two hundred years of their history made such tremendous political and economic progress, have been unable to give artistic expression to their new nation? ${ }^{44}$

Otros se preguntaban: "Was there enough American literature -or for that mattter, history- to engage the full and prolonged attention of genuine scholars?" 45 En el informe gubernamental de 1953, del que procede la cita mencionada líneas atrás, se planteaba cómo desprenderse del sambenito de "pueblo joven y sin cultura" que pesaba sobre Estados Unidos. El tema no era nuevo. Uno de los primeros en sacarlo a la palestra fue el lexicógrafo Noah Webster ya a finales del siglo XVIII. Este autor ha sido

culos norteamericanos se han expresado continuas suspicacias, cuando no desprecio y rechazo rotundo del papel de los intelectuales desde prácticamente el tiempo de los Padres Fundadores. Siguiendo esa lógica, Norteamérica necesitaba brazos fuertes, gente dinámica y activa, que trabajase, que inventase; no que pasara el día divagando o filosofando. Aunque con las cautelas necesarias, son interesantes respecto a este tipo de percepciones, los comentarios de Ramiro de Maeztu: "los albañiles ganan más que los profesores de universidad", "Los que se quedaron en colegios y universidades, exclusivamente consagrados a tareas intelectuales, no eran los mejores ni los más estimados del país". Véase: MAEzTU, 1957, pp. 15 y 95. Más recientemente, otro español también ofreció una opinión similar de Estados Unidos: Verdú, 1997, en concreto el apartado: "El odio a los intelectuales", pp. 104-116. En este mismo sentido, el Catedrático de Economía de la Universidad de Columbia, Jeffrey Sachs, denunciaba la actitud de los sectores anti-intelectuales estadounidenses de hoy en "La amenaza antiintelectual estadounidense", El Pais, Madrid, 04-X-2008, p. 30.

43 Kотек, 2003, pp. 185 y ss.

44 How American Literature Grew. Washington, D.C., 05-III-1953. NARA RG 306, Pamphlets and Leaflets, 1953-83, box 4.

45 CunLifFe, 1988, p. 18. 
considerado uno de los padres de la Americaness y del American English ${ }^{46}$, y a quien se le atribuye la frase "America must be independent in literature as she is in politics".

La cuestión no se zanjó sin más, y las discrepancias al respecto se prolongaron bastante tiempo. En buena medida por una especie de sensación de inseguridad interna en las propias capacidades del pueblo estadounidense en materia cultural. Tiziano Bonazzi ha señalado que dicha inseguridad podría entenderse como uno de los últimos episodios del proceso histórico de separación de su antigua metrópoli y de consolidación como nación independiente que Estados Unidos estaba experimentando. Algo así como un "complejo de Edipo" 47.

Quienes lideraban entonces el American Studies Movement buscaron acabar de una vez por todas con aquellas vacilaciones y responder afirmativamente a la siguiente pregunta:

Has the time come when the people of the United States can accept as a fact the maturity of a total and autonomous American culture, distinct, as Greek or Germanic culture might be thought distinct, from the cultures of other people in other parts of the world, and suitable for higher study and research on its own term $?^{48}$

No obstante, la tarea de persuadir a los ciudadanos estadounidenses más escépticos sobre la conveniencia de potenciar los American Studies en el extranjero no fue sencilla. A veces, quienes lideraban aquella empresa tuvieron que superar dificultades en el interior del propio país que no siempre se dieron cuando su actividad se desarrolló más allá de las fronteras nacionales. Así pues en determinadas ocasiones: "The policy factor which made it inadvisilbe to issue a booklet for distribution within the USA does not pertain to overseas areas"49.

No podemos olvidar que un segmento significativo de la opinión pública interna vio con cierta preocupación el recurso a la cultura para ganar la partida propagandística a los soviéticos. Ni siquiera después del ataque japonés a Pearl Harbour, la utilización sistemática de la propaganda fue aceptada por todos. De hecho, según algunos analistas, la batalla por contar con el respaldo mayoritario del pueblo estadounidense había sido la más importante de las "batallas" libradas durante la segunda guerra mundial:

The principal battleground of this war in not the South Pacific. It is not the Middle East. It is not England or Norway, or the Russian Steppes. It is American opinion 50 .

46 Las reivindicaciones en torno a la singularidad del American English y su defensa ante las críticas de quienes desde Gran Bretaña se negaban a aceptar la validez de los nuevos giros gramaticales y de la diferente pronunciación fue una de las consignas más habituales del American Studies Movement. La enseñanza del inglés se consideró siempre como paso imprescindible, cimiento para la posterior consolidación y difusión de los American Studies. Una buena aproximación a este particular se puede encontrar en MC DAVID, 1979.

47 BonAZZI, 2004, p. 61.

48 SPILLER, 1973, p. 613.

49 The Congress for Cultural Freedom. Berlín. 26-VI-1950. NARA RG 59, Bureau of Public Affairs, 1944-62, box 67.

50 Antes del ataque japonés de Pearl Harbour la maquinaria propagandística americana ya llevaba tiempo intentando convencer a su opinión pública de la necesidad de una mayor implicación en el con- 
Finalizado aquel conflicto, en determinados sectores se pensaba que había algunas líneas de la libertad de opinión y de prensa que no se deberían volver a cruzar. No estaban dispuestos a pasar por lo mismo en tiempos de paz.

\section{5. ¿CUÁNTO GASTARON UNOS Y OTROS?}

En términos generales, el arco parlamentario estadounidense reconocía el valor que tenían determinadas actividades de acción cultural para la difusión de una imagen positiva de Estados Unidos. Las mejor valoradas para la institucionalización de los American Studies eran las presentaciones de libros, las exhibiciones, los ciclos de cine, las becas de intercambio educativo ${ }^{51}$ y los programas para la enseñanza del inglés ${ }^{52}$.

Eso de partida. Después, a la hora de hablar en términos más precisos, surgía el desacuerdo. De un lado, ni todos los agentes diplomáticos estadounidenses, ni todos los representantes políticos confiaban por igual en la eficiencia de este tipo de actividades y, consecuentemente, en la necesidad de financiarlos públicamente. Del otro, no todas las actividades aludidas tenían una misma "potencialidad" a la hora de mostrar al resto del mundo las "bondades" del American way of life. Ambas objeciones influyeron de forma considerable en los fondos que se invirtieron en este tipo de asuntos, en el grado de atención prestada, y en la elección de las audiencias.

No es ocioso señalar que la financiación pública de la acción cultural exterior se vio lastrada por los condicionantes anteriores. Quienes mantienen que Estados Unidos gastó menos dinero en propaganda de lo que lo hizo la Unión Soviética, esgrimen tal dificultad como una de las razones de aquel diferencial. Según este planteamiento, los agentes de la diplomacia cultural norteamericanos habrían contado con una libertad de acción y unos presupuestos más reducidos de los que tuvieron a su disposición sus homólogos soviéticos.

No podemos olvidar, sin embargo, que estamos hablando de lo que se financió abiertamente y contó con el respaldo del parlamento americano. A esas cifras habría que añadir las importantes contribuciones de fundaciones filantrópicas, empresas y universidades privadas. En no pocos casos, la proyección cultural estadounidense en el exterior se hizo bajo auspicio exclusivo - a veces casi total- de fondos privados. Lo invertido por esos actores no gubernamentales fue determinante, por ejemplo, en la creación del Seminario de Salzburgo en American Studies. También en la puesta en funcionamiento del Bologna Center de la John Hopkins University. Por el prestigio que pronto adquirieron, ambos proyectos se convirtieron en una suerte de "cabezas de puente" para el estudio de las Humanidades y las Ciencias Sociales estadounidenses en la Europa occidental de la segunda posguerra mundial ${ }^{53}$.

flicto mundial. Contar con el respaldo interno era imprescindible para afrontar lo que se venía encima. Como muestra de aquel clima, las palabras citadas arriba de Archibald Mac Leish futuro director de la Office of War Information. Véase: MAY, 1997, p. 91.

51 Spiller, 1975, p. 6. Para el caso español véase Delgado, 2009, p. 69.

52 Más detalles sobre este particular en: Program of English-Teaching Activities Overseas. Washington, D.C., 25-VI-1956. NARA RG 59, BFS-Plans and Development, 1955-60, box 43.

53 Véanse al respecto los informes: The Financial History and Present Financial Status of the Johns Hopkins University Bologna Center. Bologna (d.s.f). AJHUB, Correspondence with SAIS, box 22; 
Saber cuál de las superpotencias invirtió más en propaganda es un aspecto a tener en cuenta si queremos conocer con certeza los entresijos de la batalla cultural que enfrentó a soviéticos y estadounidenses. Además importa conocer cómo se empleó aquel dinero, a través de qué vías, cuál fue la aportación de los "inversores no gubernamentales", las prioridades en cada momento, etc.

El dossier How we are fighting the battle for men's minds se mueve precisamente en la línea de demostrar que los soviéticos ponían la parte del león y los estadounidenses la del ratón a la hora de financiar sus respectivos programas de propaganda cultural en el exterior ${ }^{54}$. En el mismo se recopilan una serie de artículos que rezuman un cierto maniqueísmo: los soviéticos asumían abiertamente la implicación de su gobierno en un dirty business como aquel y, además, contaban con unos presupuestos mucho más elevados que los puestos a disposición de sus homólogos estadounidenses. Uno de aquellos escritos hablaba en estos términos:

Russia's propaganda machine, like all Soviet institutions, is monolithic. The Politburo itself makes propaganda policy on a day-to day basis as well as for long-term objectives. As detailed in yesterday's New York Times, Russia regards propaganda and revolutionary activity as main forces of Soviet policy, which it backs up with armed forces that are kept at peak efficiency and strength both in times of peace and war. Last year Russia and her satellites spent an estimated $\$ 1,409,000,000$ for propaganda ${ }^{55}$.

Es decir, que Moscú no sólo no ocultaba sus actividades propagandísticas, sino que además las consideraba, junto a las propiamente revolucionaras, como un pilar de su política hacia el exterior. Por su parte:

United States expenditures for propaganda are insignificant by comparison. The information and propaganda functions of the State Department alone account for $\$ 100,000,000$. A substantial sum is at the disposal of the Army and Air Force, which have units engaged in combat psychological warfare in Korea [...]. Some funds of the Central Intelligence Agency and the Mutual Security Agency might be included in this category, too, but a detailed breakdown is not available. Certain of the agencies have some non-accountable funds for undercover activities that impinge on propaganda operations ${ }^{56}$.

Esto es lo que se publicaba. Quedan, sin embargo, bastantes detalles en el aire. Lo que invertía la Unión Soviética parecía conocerse a la perfección. Se daban cifras exactas. Cifras que hacían que lo invertido -siempre según esta fuente- en los primeros años cincuenta por parte norteamericana resultase irrisorio en comparación con su antagonista soviético.

Salzburg and Understanding. Washington, D.C., 1-IX-1955. NARA RG 59, BPA, Correspondence, Memorandums, etc., 1954-55, box 1, y JoHNSON, 1963.

54 How We Are Fighting the Battle for Men's Minds. Washington, D.C., 3-V-1953. NARA RG 306, Pamphlets and Leaflets, 1953-83, box 4.

55 Communist Expenditure for Propaganda. 11-XII-1952. Dentro del dossier mencionado: How We Are Fighting the Battle for Men's Minds. Washington, D.C., 3-V-1953. NARA RG 306, Pamphlets and Leaflets, 1953-83, box 4.

56 Ibidem. Las cursivas son nuestras. 
A tenor de lo expuesto, cabe preguntarse: ¿por qué supuestamente el gasto del bloque comunista se conocía detalladamente y el propio quedaba envuelto en términos confusos, en vaguedades, tales como: "Some funds of the Central Intelligence Agency and the Mutual Security Agency", "Breakdown is not available", "Nonaccountable funds", "A substantial sum"?

Resulta extraño que el diferencial fuese tan amplio. En teoría, Estados Unidos sólo estaba invirtiendo en propaganda cultural una duodécima parte de lo que gastaba la Unión Soviética. Claro que no se mencionaban las sumas aportadas por grandes multinacionales y por fundaciones privadas norteamericanas. Ya quedó dicho que éstas colaboraron generosamente con las instancias gubernamentales en lo que se entendía como una suerte de "comunidad de intereses" 57 frente a la amenaza de extensión del comunismo.

Si así fuese, a lo mejor convendría repensar algunas de las presunciones que se han dado por buenas en parte de la bibliografía mencionada. Algunas obras han asumido, sin demasiado esfuerzo crítico, ni cotejo documental, el planteamiento de que la balanza del dinero invertido en propaganda cultural cayó siempre del lado soviético; otras han pasado de puntillas sobre el tema.

Tal vez, una cuestión que pueda aportar algo de luz sea la forma de proceder de unos y otros. Al parecer y según cuentan los informes de la diplomacia cultural americana, los soviéticos actuaban abiertamente. Era un tema considerado de gran importancia; su valía no se ocultaba y consecuentemente era abordado como uno más de los asuntos más relevantes de la actualidad política nacional. La diplomacia cultural norteamericana, por su parte, actuaba más soterradamente; no mostraba todas sus cartas y mantenía cierto recelo o temor a ser criticada y controlada, tanto por la propia justicia del país como por la opinión pública.

Probablemente por ello, las actividades propagandísticas norteamericanas en terceros países se ejecutaban con un peculiar modus operandi:

All over Europe, in free countries that have been infiltrated by Soviet Communists, hundreds of millions of anti-Communist pamphlets are circulating with messages of freedom, subverting Russia's work. These pamphlets bear the imprint of indigenous labor unions, veterans' organizations and other groups, but they were designed and written by Americans [...] The United States has learned some lessons, and in putting aside its own label, has aroused a fighting spirit among these organizations. At the same time the pamphlets, because of their local sponsorship, have become more influential in arousing doubts of Communist doctrine ${ }^{58}$.

Tampoco podemos descartar que se procediese de este modo porque se entendía que era una vía más eficiente. Que los movimientos de protesta anticomunista fuesen, aparentemente, espontáneos y de exclusiva manufactura autóctona resultaba más rentable en términos de opinión pública que si aparecía la mano de Washington detrás.

57 BERGHAHN, 1999.

58 How We Are Fighting the Battle for Men's Minds. Washington, D.C., 3-V-1953. NARA RG 306, Pamphlets and Leaflets, 1953-83, box 4. Las cursivas son nuestras. 
La empresa privada estadounidense, aparte de las universidades y las fundaciones filantrópicas, ayudó, y parece que no poco, a ganar la batalla cultural contra el comunismo ${ }^{59}$. A veces lo hizo en connivencia y de acuerdo con los mandatarios gubernamentales, $\mathrm{y}$ a veces por su cuenta. Parte de las obras que hemos citado no han prestado demasiado interés 60 a aquellas "donaciones" extra-oficiales. Hasta que no sepamos con exactitud de cuánto dinero estamos hablando es difícil sacar conclusiones precisas. Existen, no obstante, un buen número de indicios que permiten revisar las hipótesis defendidas por quienes han asumido que la Unión Soviética invirtió mucho más que Estados Unidos en propaganda cultural.

\section{Cifras de lo invertido por los soviéticos en propaganda en 1950 , según publicaba la prensa estadounidense}

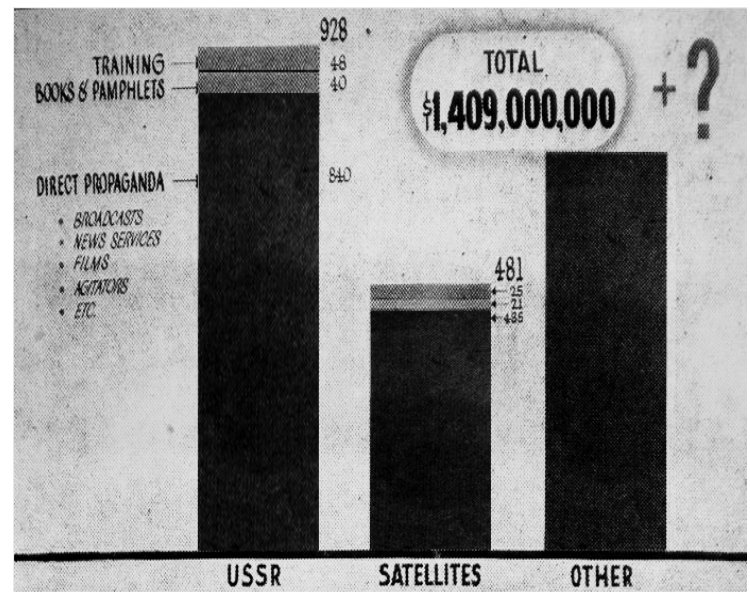

Fuente: How We Are Fighting the Battle for Men's Minds. 3-V-1953. NARA RG 306, Pamphlets and Leaflets, 1953-83, box 4.

Lo antedicho para el contexto general puede aplicarse igualmente para el tema que nos ocupa relativo al empeño gubernamental de estimular la institucionalización de los American Studies en las universidades del extranjero:

The extent of the contribution which American educational institutions with organized programs have contributed through the exchange of persons to the development of American Studies in foreign countries would appear to have been extremely significant ${ }^{61}$.

59 Veánse los documentos: How Free Enterprise Has Helped to Make America Great. Washington, D.C., 5-III-1953. NARA RG 306, Pamphlets and Leaflets, 1953-83, box 4; Current Contributions of American Private Agencies to the Development of American Studies in Foreign Countries. Washington, D.C., 13-IV-1955. NARA RG 59, BFS-Plans and Development, 1955-60, box 43 y American Business an the USIA Program Overseas. Washington, D.C., 24-III-1954. NARA RG 306, Master Budget Files, 1953-64, box 56.

60 "Histories of international relations, however, still tend to relegate foundations to the sphere of footnotes". SCHMIDT, 2004, p. 117.

61 Son varios los informes gubernamentales que inciden en la importancia de lo invertido por parte de universidades y fundaciones privadas en el sostenimiento de los American Studies. Algunos de los 


\section{ESFUERZOS PRIVADOS EN PRO DE LOS AMERICAN STUDIES}

Antes que el gobierno, fue la propia sociedad estadounidense, o más concretamente un segmento de la misma -sobre todo profesores de universidad e intelectuales- la que empezó a reivindicar la necesidad de contar con unas letras propias, con unas Humanidades y unas Ciencias Sociales centradas en el estudio y en la reivindicación de la American Culture.

Anticipando lo que después popularizó Kennedy, estas organizaciones actuaron bajo el lema: "My fellow Americans: ask not what your country can do for you-ask what you can do for your country" 62 . Un trabajo que, en general, casaba muy bien con los planes en política exterior trazados desde la Casa Blanca. La administración norteamericana permitió, salvo alguna excepción, que aquellas instituciones privadas siguiesen con sus planes, sin interferir demasiado. ¿Para qué intervenir dónde ya lo hacían aquellas? Además de más rentable económicamente, este distanciamiento tenía otras ventajas.

Resulta revelador al respecto el análisis del modus operandi de determinadas asociaciones e instituciones europeas relacionadas con Estados Unidos. Mayoritariamente optaron por mantener cierta distancia con las instancias gubernamentales de aquel país. En las reuniones anuales del Congress of European American Associations -CEAA- se aconsejaba actuar de este modo. Se advertía que de lo contrario todas las actividades que realizaban dentro de sus respectivos programas de American Studies, tales como mesas redondas y conferencias, exhibiciones de arte, conciertos, exposiciones de libros, etc., podían ser vistas con recelo por parte de cierta intelectualidad europea. Lo mejor era no hacer pública la vinculación oficial con los agentes diplomáticos norteamericanos. Para solventar este escollo y evitar la pérdida de la ayuda de Washington -que sin duda necesitaban- se articularon canales indirectos o vías privadas, alejadas lo más posible de los mecanismos oficiales. Las siguientes declaraciones resultan reveladoras del clima que vivían aquellos que por entonces tenían sus intereses vinculados, de una forma u otra, con Estados Unidos:

From conversations with officers of the CEAA and the officers and members of individual Associations, it was evident that no matter how necessary and welcome American help to the Movement would be, they prefer to receive it from American private sources (foundations, industrial firms, educational and social institutions and individuals) and not from the U.S. Government and its agencies. In cases where such governmental assistance is forthcoming, the officers and members point out that it brands them as agents of American propaganda and diminishes the value of their work ${ }^{63}$.

más notorios son: Current Contributions of American Private Agencies to the Development of American Studies in Foreign Countries. Washington, D.C.,13-IV-1955. NARA RG 59, BFS-Plans and Development, 1955-60, box 43; Establishment and Operation of Institutes for American Studies Conferences or Similar projects. Washington, D.C., 24-XII-1952; College and Universities Participation Program. 7-III-1955; Development of American Studies in foreign universities. 10-III1955 y Development of Colloquia, Seminars, Institutes and Workshops overseas.Washington, D.C.,12IV-1955. Ambos en: NARA RG 59, BFS-Plans and Development, 1955-60, box 43.

62 SAFIRE, 1997, p. 792.

63 The Congress of European American Associations. A Report of its Convention in Rome. 1 Washington, D.C., 5/20-IX-1955. NARA RG 306, Subject Numeric Files, 1953-67, box 29. Las cursivas son nuestras. 
Aquel distanciamiento permitía argüir que frente a la asfixiante atmósfera de control ideológico que ejercía Moscú sobre cualquier evento cultural, Washington, por el contrario, dejaba gran margen de libertad a las iniciativas privadas. Los "Soviet Studies" respondían a las pautas y cánones oficiales. No había lugar a la creatividad individual del autor. Los American Studies, en cambio, eran buena muestra de un talante diferente, de un gobierno menos restrictivo que dejaba hacer, que no se inmiscuía en la creatividad, ni en la obra de sus escritores, de sus pintores, etc.

La colaboración de estas organizaciones privadas con el gobierno norteamericano en la containment policy tuvo gran importancia en esta historia de la evolución de los Estudios Norteamericanos como campo autónomo en los los currículum universitarios europeos. Aquel nexo fue vital, no sólo por el dinero aportado, sino también porque significaba una magnífica oportunidad para que el aparato diplomático estadounidense no apareciese detrás de ciertas iniciativas. Las fundaciones daban la cara, tenían mejor prensa en Europa y no estaban "contaminadas" con el estigma de ser instrumentos propagandísticos. El elenco de las que participaron en la financiación de programas para la enseñanza del inglés o para el sostenimiento de seminarios, cursos o exhibiciones en el ámbito de los Estudios Norteamericanos fue amplio.

La lista que adjuntamos a continuación, procedente del Bologna Center de la Johns Hopkins University ${ }^{64}$, no es más que una aproximación sin ánimo de exhaustividad, ya que sin duda fueron bastante más las instituciones y compañías que financiaron la promoción y difusión de los American Studies en el viejo continente. Pensaban de este modo contribuir a la consolidación de un bloque atlantista que ahogase cualquier posibilidad de extensión del telón de acero más allá de su demarcación inicial.

Parece, pues, fuera de toda duda que los American Studies se beneficiaron de la generosidad de estos modernos "mecenas de las artes". Fueron muchos los programas de enseñanza del inglés, de literatura, historia, ciencia política y otras materias sobre la realidad socio-cultural estadounidense que fueron financiados en parte, o en algunos casos íntegramente, con dinero de estas fundaciones filantrópicas ${ }^{65}$. En España, por ejemplo, la Fundación Ford sufragó un amplio número de proyectos para la formación de profesores de inglés a partir de mediados de los sesenta y, en especial, desde los primeros años setenta ${ }^{66}$.

También se financiaron otras actividades como donaciones de libros a bibliotecas $^{67}$, se sufragaron viajes de profesores europeos a Estados Unidos, conferencias,

64 The Congress for Cultural Freedom. Berlín. 26-VI-1950. NARA RG 59, Bureau of Public Affairs, 1944-62, box 67.

65 SCHMIDT, 2004, pp.124-125.

66 Véanse los documentos: I Seminario para profesores de inglés. Valladolid, 1-V-1969. Correspondencia general de la Comisión Fulbright-España. AGA, caja 54/10571 y Presentación de Peter Fraenkel, representante de la Fundación Ford en España. 13-X-1971. ACFE, caja 4.

67 El apoyo no era algo nuevo. Sirva como ejemplo la importante donación que realizaron la Carnegie y la Rockefeller Foundation para la creación de la importante American Library de París ya en 1918. Véase: Asselineau - Copans, 1975, p. 65. 
seminarios y un largo etcétera. La cosa venía de lejos. A modo de ilustración, cabe decir que en Madrid la filantropía estadounidense coadyuvó, a finales del siglo XIX, a la financiación del Instituto Internacional, conocido popularmente como "las Bostonianas ${ }^{68}$.

\section{Organizaciones privadas que contribuyeron a la promoción de los American Studies en el bloque europeo occidental, 1945-66}

\begin{tabular}{|c|c|}
\hline Fundaciones Filantrópicas & Compañías \\
\hline Ford Foundation & American Home Products \\
\hline Rockefeller Foundation & American Machine \& Foundry \\
\hline Smithsonian Foundation & Campbell Soup Company \\
\hline Carnegie Corporation of New York & Coca-Cola Company \\
\hline Gulbenkian Foundation & Colgate Palmolive \\
\hline Arthur I. Johnson Foundation & Continental \\
\hline W.K. Kellogg Foundation & Illinois National Bank \& Trust Companny \\
\hline Max Kade Foundation, Inc & Federal-Mogul Corporation \\
\hline Aaron E. Norman Fundation & First National City Bank \\
\hline Overbrook Foundation & Ford Motor Company \\
\hline Amaricen Metal Climax Foundation & General Telephone \& Electronics \\
\hline Conrad I. Hilton Foundation & W.R. Grace \& Company \\
\hline \multirow[t]{5}{*}{ Cities Service Foundation. } & Honeywell, Inc. \\
\hline & Hark, Sharp \& Dohme \\
\hline & Quaker Oats Company \\
\hline & Trans World Airlines \\
\hline & Chicago Bridge Iron Company \\
\hline
\end{tabular}

Fuente: "Financial Status of the John Hopkins Bologna Center" 29-IV-1966. FFA. R-0679.63-139. (Elaboración propia)

No sólo eso. El propio Salzburg Seminar, que estuvo en el origen de la creación de European Association for American Studies -EAAS-, recibió una cuantiosa beca por parte de la Rockefeller Foundation ${ }^{69}$. Era la manera que tuvieron los técnicos de aquella fundación de animar a los pocos europeos que por entonces se empeñaban en estrechar la brecha atlántica. La forma más habitual de actuar fue establecer convenios de colaboración directamente con centros y universidades norteamericanas que operaron como anfitrionas. Éstas ofrecieron los cursos, gestionaron los viajes y las

68 ZULUETA, 1993. Sobre la financiación de programas de estudios, actividades culturales, creación de bibliotecas e institutos, etc., por parte de fundaciones estadounidenses pude verse: GEMELLI - MAC LEOD, 2003.

69 American Studies Project for Foreign Scholars. Washington, D.C., 1-IX-1955. NARA RG 59, BPA, Correspondence, Memorandums, etc., 1954-55, box 1. 
estancias de los europeos visitantes. A cambio recibieron sustanciosas donaciones por parte de las distintas asociaciones filantrópicas ${ }^{70}$.

Hubo más. También se puso dinero para la creación de cátedras específicas sobre American Studies en algunas universidades europeas. Un ejemplo destacado fue el de la Universidad Libre de Berlín ${ }^{71}$. Esta institución recibió importantes aportaciones monetarias por parte de la Ford Foundation para el desarrollo de programas relacionados con el estudio de la realidad norteamericana. Alemania occidental -también, aunque en menor medida, Japón ${ }^{72}$ - fue uno de los focos prioritarios de aquellas iniciativas filantrópicas. No en vano estaba en la primera línea de batalla frente al bloque comunista. El interés porque este tipo de iniciativas se consolidase allí fue grande $^{73}$. Si funcionaban correctamente se convertirían en el espejo perfecto para captar la mirada de la parte oriental y mostrar las ventajas del modelo americano frente al comunista. El altruismo de aquel tipo de colaboración se vio fuertemente politizado por la política de contención del comunismo que se marcaba desde Washington.

En términos generales, no se trató de imposición por parte del gobierno norteamericano, sino que la mayoría de los gestores culturales de aquellas fundaciones asumieron como propios los planes geopolíticos trazados por la diplomacia cultural de Washington. A veces, sucedió a la inversa. Los planes diseñados por instituciones privadas fueron adoptados y financiados por organismo públicos.

La estrecha vinculación entre las dos esferas se mantuvo hasta finales de la década de los sesenta. En 1967 la revista Ramparts publicaba un artículo en el que se denunciaban las conexiones secretas y la financiación que había recibido la National Student Association(NSA) por parte de la CIA. El escándalo desatado por el descubrimiento de la implicación de la CIA en actividades culturales salpicó también al mundo de la filantropía estadounidense ${ }^{74}$. En adelante, las grandes fundaciones Ford, Rockefeller, Carnegie, etc., y buena parte de las corporaciones señaladas intentaron desvincularse de aquellas actividades propagandísticas del gobierno de Estados Unidos ${ }^{75}$. Su nombre y reputación se vieron seriamente manchados por aquella colaboración ${ }^{76}$.

70 Ibidem.

71 The Free University's request for Funds From the Ford Foudation. Washington, D.C., 20-IV1958. NARA RG 59, BFS- Plans and Development, 1955-60, box 44.

72 Alemania occidental se convirtió, junto a Japón, en una de las zonas donde más programas de American Studies se pusieron en marcha en los primeros años cincuenta. Dentro de las distintas medidas de "reeducación" de estos dos países, se prestó gran atención a la difusión de aquel tipo de estudios por la potencialidad en la transmisión de valores democráticos que se les atribuía. Véase: American Institutes and Seminars in Europe. A preliminary list, prepared for the American Studies Association. Washington, D.C., 24-IX-1970. LC-ASA, Part I: Administrative File, 1946-2003, box I: 111.

73 Que sepamos, no existen estudios monográficos sobre el desarrollo de los American Studies en las universidades alemanas después de la segunda guerra mundial. Un informe muy interesante al respecto es: American Studies in German Universities" en "ASA correspondence with different American Associations. Washington, D.C., 22-X-1951, LC-ASA, archives Part I: Administrative File, 1946-2003, box I: 22 .

74 JEFFREYS-JONES, 1986, p. 122.

75 BERGHAHN, 1999, pp. 399 y ss.

76 Tal "comunidad de intereses" tuvo consecuencias bastantes negativas para la imagen en Europa de algunas de aquellas entidades privadas. Hoy en día no es raro que las manifestaciones de grupos antisistema, anarquistas o similares acaben con la quema de los emblemas, banderas o incluso las instala- 
Lo sucedido posteriormente escapa a la acotación temporal asumida en este artículo. No obstante, se puede adelantar que el asunto no quedó ahí. Muy probablemente, las alianzas entre actores privados y públicos estadounidenses en la batalla cultural contra Moscú continuaron. Si bien cabe suponer, con unas características diferentes, ya que el contexto general de la Guerra Fría también había cambiado.

\section{REFERENCIAS BIBLIOGRÁFICAS}

Asselineau, Roger - Copans, Simon

1975 "American Studies in France: the new face of a tradition". En WALKER, Robert (ed.). American Studies Abroad. London. Greenwood Press, pp. 53-57.

BERGHAHN, Volker

1999 "Philanthropy and Diplomacy in the American Century". Diplomatic History. Boulder. no 23, pp. 393-419.

BonAzZI, Tiziano

2004 "Not like us: il controcanto americano all'antiamericanismo europeo". En CRAVERI - Quagliariello (eds.), pp. 45-72.

Craveri, Piero - Quagliariello, Gaetano (eds.).

2004 L'antiamericanismo in Italia e in Europa nel secondo dopoguerra. Catanzaro. Rubbettino Editore.

CUNLIFFE, Marcus

1998 "Problems and Tendencies in American Studies". En García Diez, Enrique (ed.). American Studies in Spain. Valencia. Tirant lo Blanch. pp. 11-20

DELGADO, Lorenzo

2009 "Viento de Poniente". El programa Fulbright en España. Madrid. LID Editorial Empresarial.

FULBRIGHT, William

1972 The Crippled Giant-American Foreign Policy and its Domestic Consequences. New York. Vintage Books.

Gemelli, Giuliana - MAC LeOD, Roy (eds.)

2003 America Foundations in Europe. Grant-Giving Policies, Cultural Diplomacy and Trans-Atlantic Relations, 1929-80. Bruselas. P. I. EPeter Lang.

Gienow-HeChT, Jessica

1999 Transmission Impossible. American Journalism as Cultural Diplomacy in Postwar Germany, 1945-1955. Baton Rouge. Louisiana State University Press.

2000 "Shame on Us? Academics, Cultural Transfer, and the Cold War - A Critical Review". Diplomatic History. Boulder. vol. 24. n 3, pp. 465- 494.

ciones de algunas compañías de Estados Unidos. Las fundaciones no han escapado a esta asociación y han sido también objeto de algunos ataques, no sólo en la actualidad sino desde hace tiempo. Véase: Ford Foundation Under Attack. Washington, D.C., 10-VII-1970. NARA RG 59, Subject Numeric Files, 1970-73, box 387. 
KENNEDY, Liam -LuCAS, Scout

2005 "Enduring freedom: Public Diplomacy and U.S. Foreign Policy". American Quarterly. Baltimore. $\mathrm{n}^{\circ}$ 57: 2, pp. 309-310.

KоTEK, Joël

2003 "Youth organisations as a battlefield in the Cold War". En ScotT-SмiтH, Giles - KrabBendam, Hans (eds.). The cultural Cold War in Western Europe, 1945-1960. London - Portland. OR, F. Cass, pp. 168-191.

Hilton, Sylvia - VAn Minnen, Cornelis (eds.)

2007 Teaching and Studying U.S History in Europe: Past, Present and Future. Amsterdam. VU University Press.

IKENBERRY, John

2001 “America's Imperial Ambition”. Foreign Affairs. New York. vol. 81. n 5, pp. 44-60.

http://dx.doi.org/10.2307/20033268

JEFFREYS-JONES, Rhodri

1986 "The CIA and the Demise of anti-Anti-Anti-Americanism: Some Evidence and Reflexions". En Kroes, Rob - VAN Rossem, Maarten (eds.). Anti-Americanism in Europe. Amsterdam. Free University Press, pp. 121-136.

JOHNSON, Walter

1963 American Studies Abroad: progress and difficulties in selected countries. Washington. D. C. U. S. Advisory Commission on International Educational and Cultural Affairs. Government Printing Office.

LefFLer, Melvyn - PAINTER, David (eds.)

1994 Origins of the Cold War: an International History. London. Routledge. LeÓn Aguinaga, Pablo

2010 Sospechosos habituales: Hollywood, Estados Unidos y la España de Franco, 1939/1960. Madrid. CSIC.

MAEZTU, Ramiro

1957 Norteamérica desde dentro. Madrid. Editora Nacional.

MAIER, Charles (ed.)

1996 The Cold War in Europe: Era of a Divided Continent. Princeton. Markus Wiener.

MAY, Elaine

1996 "The Radical Roots of American Studies". American Quarterly. Baltimore. $n^{\circ} 48$, pp. 179-200.

MAY, Lary

1997 "Made for Export: Hollywood and the Creation of Cold War Americanism, 1940-58". En BlaIR, John G. - WAGNLEITNER, Reinhold (eds.). Empire: American studies (Selected Papers from the Bi-National Conference of the Swiss and Austrian Associations for American Studies at the Salzburg Seminar). Tübingen. Gunter Narr Verlag, pp. 91-121.

MC DAVID, Raven

1979 "American English: a bibliographic essay". American Studies International. Washington, D.C. vol. 17, pp. 3-45. 
NiNKOVICH, Frank

1996 U.S. Information Policy and Cultural Diplomacy. New York. NY Foreign Policy Association.

NiÑO, Antonio

2009 "Uso y abuso de las relaciones culturales en la política internacional".

PELLS, Richard Ayer. Madrid. nº 75: 3, pp. 25-61.

1997 Not Like Us. How Europeans Have Loved, Hated and Transformed American Culture Since World War II. New York. Basic Books.

1989 The Liberal Mind in a Conservative Age. New York. Paperback.

RODRÍGUEZ JIMÉNEZ, Francisco Javier

2010 ¿Un «antídoto» contra el antiamericanismo? American Studies en España, 1945-69. Valencia. Publicacions de la Universitat de València.

SAFIRE, William

1997 Lend me your ears: great speeches in History. New York. Norton.

ScOTT-SMith, Giles

2007 "Laying the Foundation: U.S. Public Diplomacy and the Promotion of American Studies in Europe". En HiLTON - VAN MinNen (eds.), pp. 47-61.

2008 Networks of Empire: The US State Department's Foreign Leader Program in the Netherlands, France, and Britain 1950-1970. Bruselas. Peter Lang.

SCHMIDT, Oliver

2004 "Small Atlantic World: U.S. Philanthropy and the Expanding International Exchange of Scholars after 1945". En GIENOW-Hecht, Jessica - Schumacher, Frank (eds.). Culture and international history. Oxford. Berghahn books, pp. 135-156.

SKARD, Sigmund

1958 American Studies in Europe: Their History and Present Organization. Philadelphia. University of Pennsylvania Press.

Soutou, Georges

2001 La guerre de Cinquante Ans. Les relations Est-Ouest 1943-1990. Paris. Fayard.

SNOW, Nancy

2002 Propaganda, Inc. Selling America's Culture to the World. New York. Paperback.

SPILLER, Robert

1973 "Unity and Diversity in the Study of American Culture: The American Studies Association Perspective". American Quarterly. Baltimore. ${ }^{\circ}$ 25, pp. 611-618. http://dx.doi.org/10.2307/2711699

1975 "The Fulbright Program in American Studies Abroad: Retrospect and Prospect”. En WALKER, Robert (ed.). American Studies abroad. London. Greenwood Press, pp. 3-9. 
STONOR SAUNDERS, Frances

2001 La CIA y la Guerra Fría cultural. Madrid. Debate.

VÁZQueZ MONTALBÁn, Manuel

1973 La penetración norteamericana en España. Madrid. Cuadernos para El Diálogo.

VERDÚ, Vicente

1997 El Planeta Americano. Barcelona. Anagrama.

VERHEUL, Jaap (eds.)

1999 Through the Cultural Looking Glass: American Studies in Transcultural Perspective. Amsterdam. VU University Press.

ZASLAVSKY, Victor

2004 "L'antiamericanismo organizzato nell'Unione Sovietica staliniana". En Craveri - Quagliariello (eds.), pp. 85-106.

Zulueta, Carmen de

1993 Ni convento ni college: la Residencia de Señoritas. Madrid. Residencia de Estudiantes. 